\title{
Phyto-reduction of graphene oxide using the aqueous extract of Eichhornia crassipes (Mart.) Solms
}

\author{
M. Jannathul Firdhouse $\cdot$ P. Lalitha
}

Received: 7 July 2014 / Accepted: 2 September 2014/Published online: 8 October 2014

(c) The Author(s) 2014. This article is published with open access at Springerlink.com

\begin{abstract}
The aqueous extract of Eichhornia crassipes was used as reductant to produce graphene from graphene oxide by refluxing method. The complete reduction of graphene oxide was monitored using UV-Vis spectrophotometer. Characterization of graphene was made through FTIR, XRD, and Raman spectroscopy analysis. The stability of graphene was studied by thermal gravimetric analysis and zeta potential measurements. The nature and surface morphology of the synthesized graphene was analyzed by transmission electron microscopy. The production of graphene using phytoextract as reductant emphasizes on the facile method of synthesis and greener nanotechnology.
\end{abstract}

Keywords Graphene $\cdot$ FTIR $\cdot$ SEM $\cdot$ Raman spectroscopy $\cdot$ TEM

\section{Introduction}

Nanotechnology provides a new platform to innovations in all fields of research in order to meet the challenges towards green environment. Nanomaterials have been integrated into numerous applications in electronics, sensors, and biomedical fields because of its range of diverse properties. Nanoscience helps to reduce the formation and emission of pollutants, which helps in the betterment of technological processes involved in research field. The

\section{J. Firdhouse · P. Lalitha $(\bowtie)$}

Department of Chemistry, Avinashilingam Institute for Home

Science and Higher Education for Women University,

Coimbatore 641043, Tamil Nadu, India

e-mail: goldenlalitha@gmail.com

M. J. Firdhouse

e-mail: kfirdhouse@gmail.com rapid development of nanotechnology has led to the commercial applications which enhance the use of a great variety of manufactured nanoparticles. Nanoparticles can be grouped into four types as carbon-based nanomaterials, metal nanoparticles, denderimers, and nanocomposites [14]. Carbon-based nanomaterials hold credit for the innumerable applications in all fields of science and technology. One of the carbon-based nanomaterial of great concern in recent years is graphene.

Graphene has become a fascinating material due to its novel structural, electronic, thermal, and mechanical properties which arise from the collective behavior of electrons. Graphene was discovered in 2004 from graphite by a simple process but of late its significance has attracted the researchers in the field of material science. Its unique optical properties find potential applications as photovoltaics and transparent conductors. Reduced graphene oxide is also used in nanoelectronics, sensors, nanocomposites, and in water treatment. Graphene is also considered as a promising material for biomedical applications due to its surface enhanced Raman scattering, aqueous processability, and surface functionality. In cancer therapies, graphene is used as potential nanocarrier drugs in gene delivery and bio-imaging purposes [5-13]. Few layer graphenes are used as flexible transparent conductors and to reduce wear and friction on sliding steel surfaces. Among the diverse methods available for synthesis of graphene, the most popular one is chemical synthesis.

The chemical reduction of graphene oxide using hydrazine as reducing agent is the successful method used for the production of graphene. One of the drawbacks of this method is that hydrazine hydrate is highly toxic and explosive in nature. This necessitates milder conditions of preparation of graphene. A simple approach was adopted by Zhang et al. using L-Ascorbic acid as reducing agent in 
the preparation of graphene [14] and its solubility was also studied on common solvents such as water, $\mathrm{N}, \mathrm{N}$-dimethylformamide and $N$-methyl-2-pyrrolidone [15]. Ma et al. have prepared a stable suspension of reduced graphene oxide (RGO) using L-Lysine and carboxymethyl starch as a stabilizing agent [16]. In view of the varied applications of graphene and the requisite to disembark newer methods of synthesis of graphene, this research work is aimed at biosynthesis of graphene using plant extracts.

Eichhornia crassipes (Mart.) Solms is a free floating aquatic plant well known for its production abilities and removal of pollutants from water. The presence of proteins, carbohydrates, alkaloids, quinines, anthocyanins, and several other metabolites in its extracts is reported [17]. Recently, rapid synthesis of gold nanoparticles using aqueous extract of $E$. crassipes (Water Hyacinth) portrays its use in nanoparticle synthesis [18]. In the present work, a facile method of reduction of graphene oxide was carried out using E. crassipes extract. The reduced graphene oxide was characterized by UV-Vis spectroscopy, Raman spectroscopy, FTIR spectroscopy, and XRD and TEM analysis.

\section{Experimental}

\section{Materials}

Chemicals and reagents used in this study were of analytical grade. Graphite powder was purchased from Loba chemicals. The E. crassipes were collected from the local water body in Coimbatore.

\section{Preparation of graphene oxide}

Graphene oxide was prepared by modified Hummer's method. Graphite powder ( $2 \mathrm{~g})$ was treated with concentrated sulphuric acid (46 ml) in ice-cold condition and stirred for $2 \mathrm{~h}$. Potassium permanganate $(6 \mathrm{~g})$ was added in regular intervals of $30 \mathrm{~min}$ at temperature $35^{\circ} \mathrm{C}$ and the mixture was stirred continuously in an oil bath for $2 \mathrm{~h}$ at $50{ }^{\circ} \mathrm{C}$. Doubly distilled water $(92 \mathrm{ml})$ was added with vigorous stirring for 15 min till dark brown suspension was obtained. Hydrogen peroxide $30 \%(280 \mathrm{ml})$ was added until the solution turned pale yellow. The solution was filtered with $10 \%$ hydrochloric acid and finally with double distilled water to obtain pure graphene oxide. Thus, the obtained graphene oxide was homogenized using an Ultrasonic homogenizer and dried in vacuum to get few layer nanographene.

Preparation of the plant extract

Eichhornia crassipes was collected from Singanallur boat house, Coimbatore. The root portion was cut off, washed thoroughly to free it from debris and was shade dried for 20 days. The dried material was sliced and ground. Water hyacinth $(1.5 \mathrm{~kg})$ was defatted twice with petroleum ether $(2.5 \mathrm{~L})$ for $6 \mathrm{~h}$. The solvent was filtered off and the plant residue of Water hyacinth $(20 \mathrm{~g})$ was extracted thrice for $1 \mathrm{~h}$ with distilled water. The solvent was filtered, concentrated in a rotary vacuum evaporator (Equitron), and dried to get aqueous extract. Aqueous extract of E. crassipes $(100 \mathrm{mg})$ was sonicated with $100 \mathrm{ml}$ of double distilled water at $50{ }^{\circ} \mathrm{C}$ by sonic bath (Ultrasonics $-1.5 \mathrm{~L}(\mathrm{H})$ ) for $30 \mathrm{~min}$. The extract was filtered using Whatmann filter paper and refrigerated.

Synthesis of reduced graphene oxide

Graphene oxide $(180 \mathrm{mg})$ was sonicated with $360 \mathrm{ml}$ of double distilled water for 30 min to obtain a stable suspension. The stable solution was treated with $30 \mathrm{ml}$ of aqueous extract of $E$. crassipes and refluxed.

Characterization of graphene

UV-Vis spectral analysis was performed using Double beam spectrophotometer- 2202 (Systronics). X-ray diffraction analysis was carried out on X-pert X-ray diffractometer. FTIR spectral measurements were analyzed on Shimadzu Nicolet 6700 FTIR spectrometer with the resolution of $0.2 \mathrm{~cm}^{-1}$. Thermo-gravimetric analysis was carried out under nitrogen flow using SII-6300-TG-DTA (Exstar) and their masses were recorded as a function of temperature. The samples were heated from room temperature to $600{ }^{\circ} \mathrm{C}$ at $5{ }^{\circ} \mathrm{C} / \mathrm{min}$. The zeta potential value was measured by NanoPartica SZ 100 (Horiba). The Raman spectrum was obtained using R-3000 QE with an optical resolution of $6 \mathrm{~cm}^{-1}$. The TEM images of reduced graphene oxide were analyzed by FEI's Tecnai ${ }^{\mathrm{TM}} \mathrm{G} 2$ transmission electron microscope.

\section{Results and discussion}

Graphene oxide was treated with aqueous extract of $E$. crassipes and refluxed continuously for $10 \mathrm{~h}$. After $6 \mathrm{~h}$, the brown suspension turned black followed by precipitation. The production of graphene was confirmed by UV-Vis spectrophotometer.

UV-Vis spectroscopy

The UV-Vis spectrum shows an absorption peak at $234 \mathrm{~nm}$ for graphene oxide corresponding to the $\pi-\pi^{*}$ transition of aromatic $\mathrm{C}-\mathrm{C}$ bonds. The absorption peak for graphene at $274 \mathrm{~nm}$ confirms the reduction of graphene oxide (Fig. 1) 


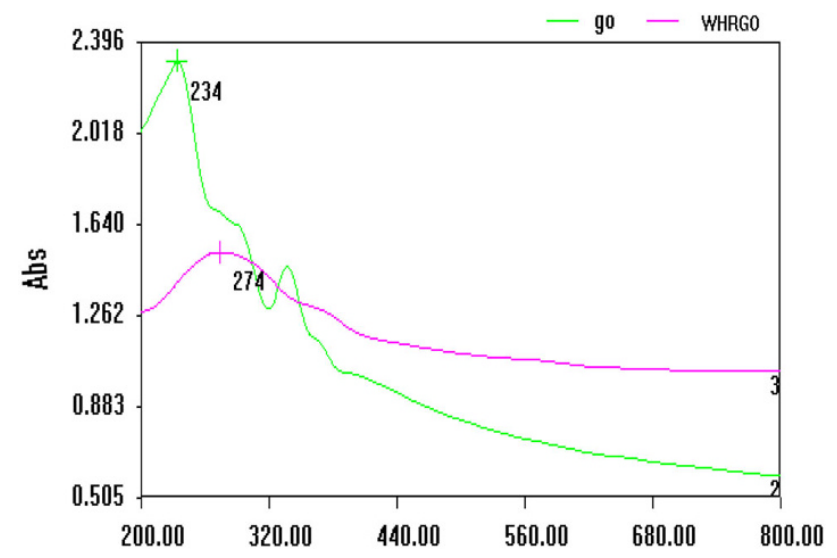

Fig. 1 UV-Vis spectra of graphene oxide and synthesized graphene (WHRGO)

with the intensity of graphene oxide being more than the synthesized graphene. The occurrence of red shift has been used as a monitoring tool for the reduction of graphene oxide. The flat absorption band due to dispersion of Dirac electrons in graphene is responsible for the wide band applications.

FTIR analysis

Figure 2 represents the FTIR spectra of graphene oxide and graphene. The peaks at 1,720 and $1,610 \mathrm{~cm}^{-1}$ are assigned to carbonyl and aromatic region, respectively, for graphene oxide. The absence of carbonyl peak in the FTIR of graphene indicates the formation of reduced graphene oxide.

\section{$\mathrm{XRD}$ analysis}

The XRD patterns of graphene oxide (Fig. 3) show a sharp and intense peak at $2 \theta=9.75^{\circ}$ corresponding to the

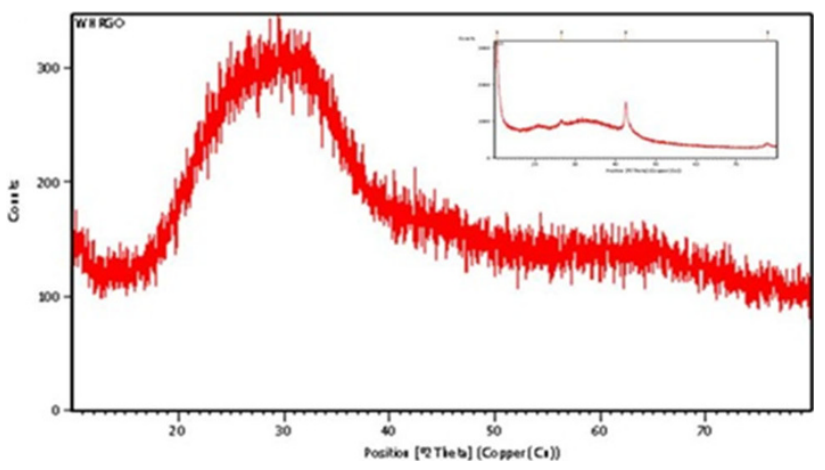

Fig. 3 XRD pattern of reduced graphene oxide; Inset XRD pattern of graphene oxide

Bragg's reflection of (002) with an interlayer distance of $7.6 \AA$. After the reduction of graphene oxide, the peaks at $2 \theta=26^{\circ}$ correspond to the Bragg's reflections of $(001)$ and the disappearance of the peak at $9.75^{\circ}$ confirms the formation of graphene.

Thermo gravimetric analysis

Thermal stability of the graphene synthesized using the aqueous extract of E. crassipes (WHRGO) was compared with that of the chemically synthesized graphene (CRG) by TGA (Fig. 4). A slight mass loss below $100^{\circ} \mathrm{C}$ can be attributed to the loss of adsorbed water in both $\mathrm{CRG}$ and WHRGO samples. CRG exhibits $10 \%$ weight loss up to $250^{\circ} \mathrm{C}$ whereas as WHRGO possesses $15 \%$. The significant mass loss is detected when WHRGO is heated up to $550^{\circ} \mathrm{C}$ but in graphene oxide, the pyrolysis of the remaining oxygen-containing groups and also the burning of ring carbon were observed. The enhanced thermal stability is due to the removal of oxygen-containing functional
Fig. 2 FTIR spectra of synthesized graphene (WHRGO) and graphene oxide (GO)

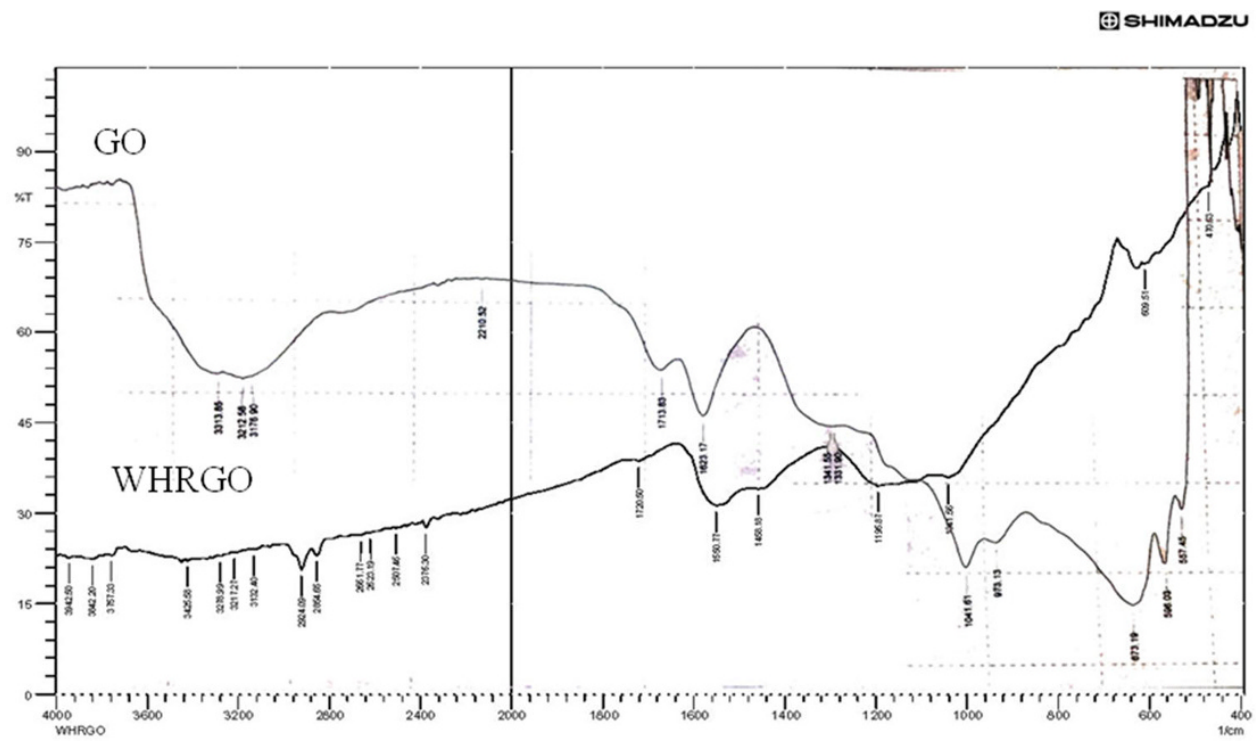


Fig. 4 TGA-thermogram of phyto-reduced graphene oxide WHRGO and chemically synthesized graphene $(\mathrm{CRG})$

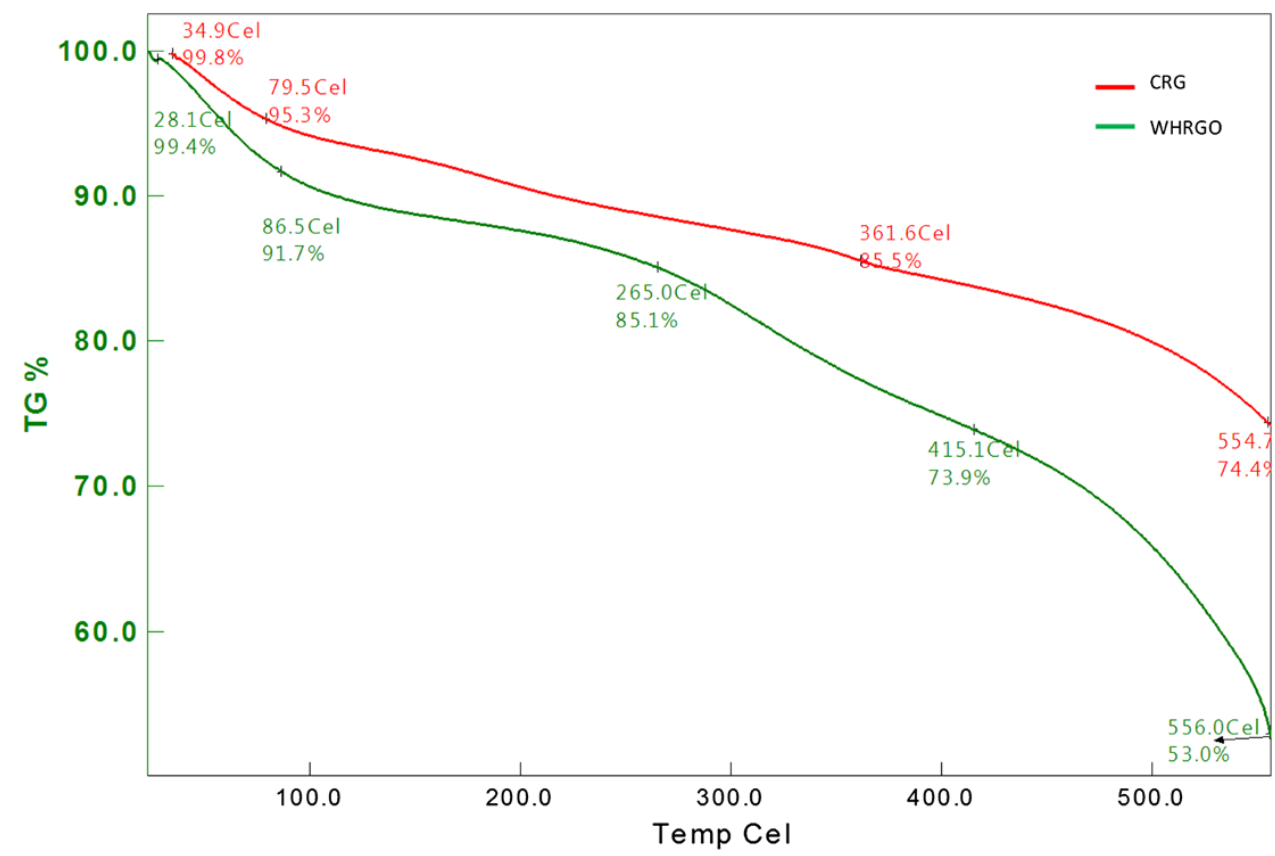

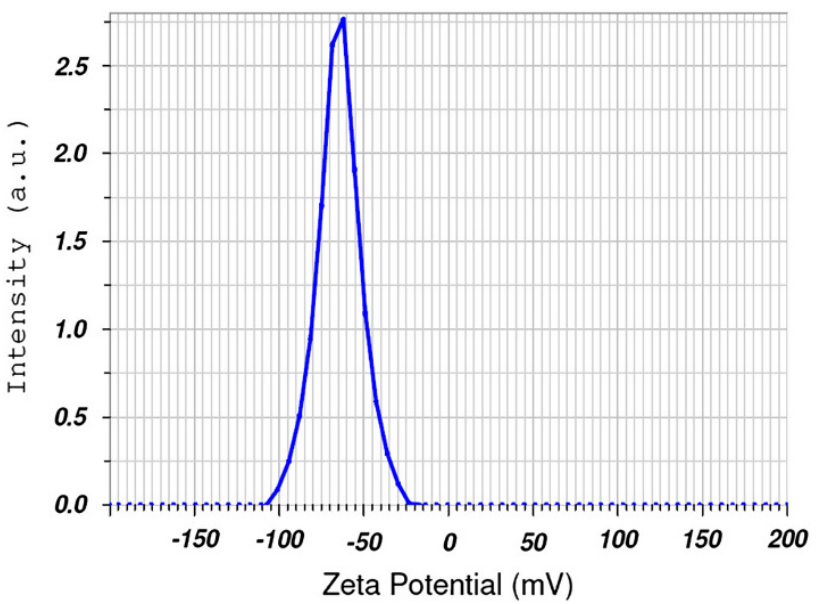

Fig. 5 Zeta potential measurements of synthesized grapheneWHRGO

groups. This reveals the stability of the synthesized graphene and confirms the degree of reduction.

\section{Zeta potential analysis}

The zeta potential measurement of the synthesized graphene using the aqueous extract of E. crassipes is given in Fig. 5. The value of zeta potential of graphene $-64 \mathrm{mV}$ indicates the stability of the graphene particles. The electrical potential of the interface between the aqueous solution and the stationary layer of ions attached to the particle is used to characterize the electrical properties of the particles. The zeta potential of reduced graphene dispersion is $\mathrm{pH}$ dependent and is also lower than that of graphene oxide. Graphene possessing positive surface charge absorbs anions, whereas

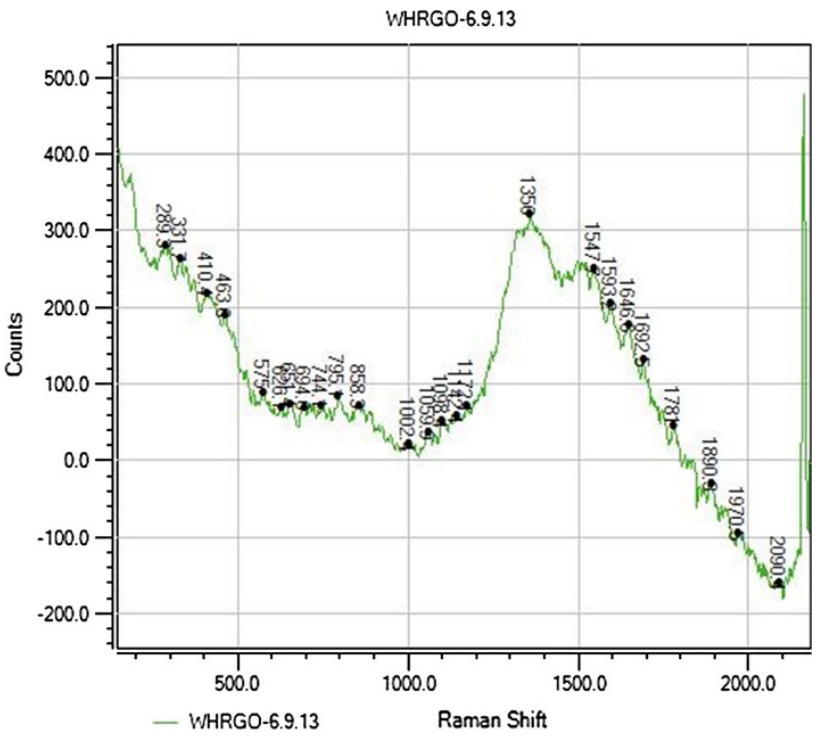

Fig. 6 Raman spectra of synthesized graphene-WHRGO

negative surface charge will have the benefits of absorbing cations [19]. Li et al. reported that the presence of surface negative charge on graphene materials attracts the positively charged $\mathrm{Cu}^{2+}[20]$. The zeta potential results of synthesized graphene (WHRGO) revealed the presence of negative surface charge which can absorb cations and may find application in the removal of heavy metal ions.

\section{Raman spectroscopy}

Figure 6 shows the Raman spectra of the synthesized graphene using the aqueous extract of E. crassipes. The 
Fig. 7 TEM images of reduced graphene oxide-WHRGO

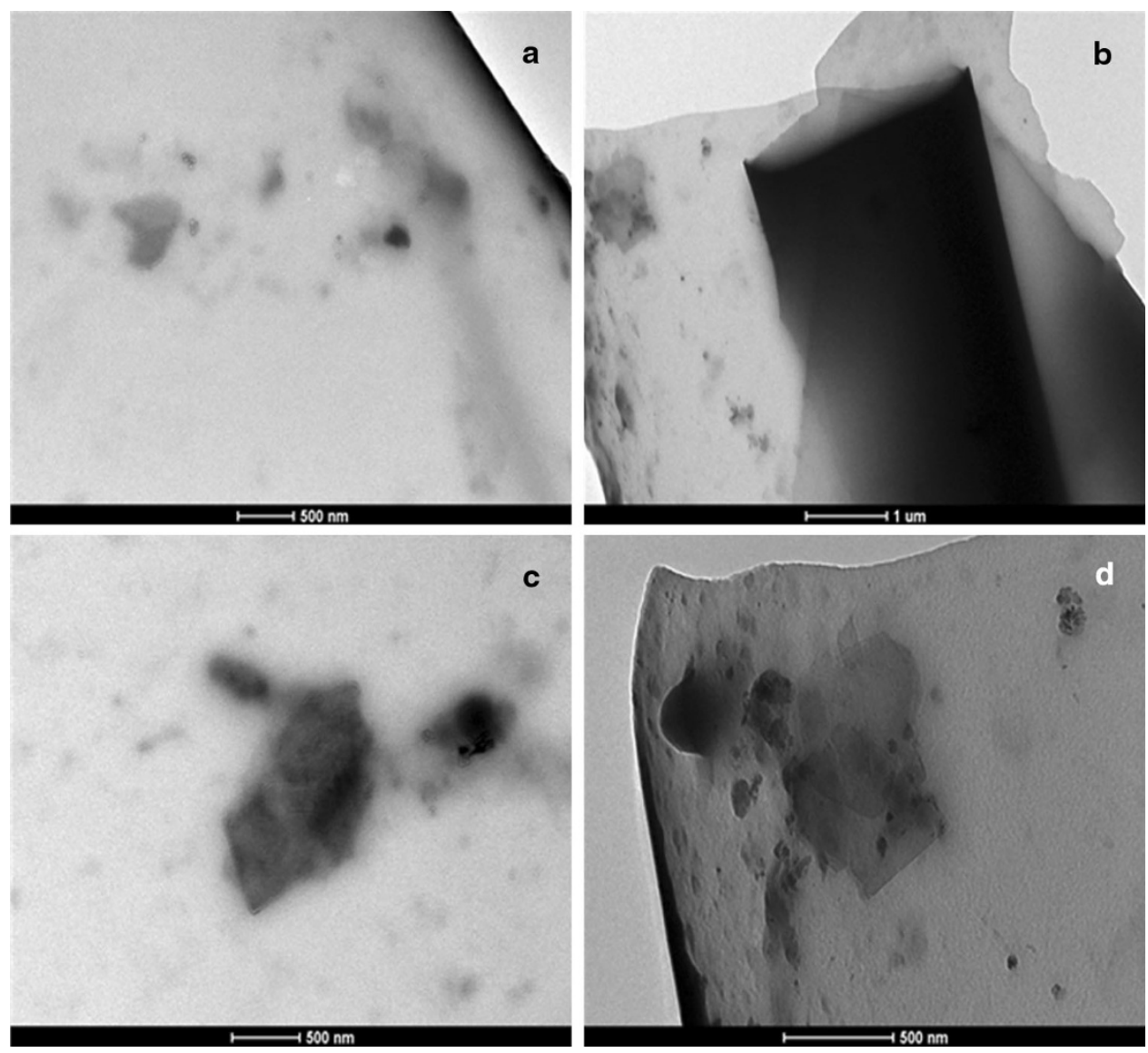

two most intense features of graphene are the $G$ peak at $1,580 \mathrm{~cm}^{-1}$ and a band at $2,700 \mathrm{~cm}^{-1}$ designated as Ǵ. The $G$ peak in graphene is due to the doubly degenerate zone center $E_{2 g}$ mode and $G$ or $2 D$ is the second order of zone boundary phonons. The G' band is normally not observed in the first order Raman spectra of defect free graphite. These phonons will give rise to a peak at $1,350 \mathrm{~cm}^{-1}$ called D peak. In Fig. 6, the peak at 1,350 and $1,594 \mathrm{~cm}^{-1}$ revealed the presence of $\mathrm{D}$ and $\mathrm{G}$ band. The absence of 2D peak confirms the formation of few layers graphene. Raman spectra provide data to make a distinction between single and few layer graphene.

\section{TEM analysis}

TEM image of the reduced graphene oxide is shown in Fig. 7. The transmission electron microscopic images (Fig. 7a, b) showed graphene sheets to be stacked on one another. Figure $7 b$, d clearly show the crystal planes of graphene with folded and flat structure, some with microwrinkling. The folding process may depend on the presence of interlayer partial chemical and hydrogen bonds. Thus the synthesized graphene in this work might contain one to few-layer sheets.

\section{Conclusion}

Reduction of graphene oxide was achieved using the aqueous extract of $E$. crassipes. The formation of reduced graphene oxide was confirmed and characterized by UV, FTIR, TEM, Raman, TGA, and XRD analysis. The zeta potential value of the synthesized graphene was found to be $-64 \mathrm{mV}$ and the thermo gravimetric analysis revealed the stability of biosynthesized graphene compared to that of chemically synthesized graphene. The shift values in Raman spectra confirm the formation of few layer graphene. The nature and surface morphology of the synthesized graphene was found to be of few layers with a wrinkling flat surface.

Acknowledgments The authors are sincerely thankful to Avinashilingam Institute for Home Science and Higher Education for Women, Coimbatore for providing research facilities to carry out this work and Department of Nanoscience and technology, Tamil Nadu Agricultural University, Coimbatore for recording Raman spectra, zeta potential, and for TEM analysis.

Conflict of Interests The authors declare that there is no conflict of interests.

Open Access This article is distributed under the terms of the Creative Commons Attribution License which permits any use, 
distribution, and reproduction in any medium, provided the original author(s) and the source are credited.

\section{References}

1. Martin, C.R., Kohli, P.: The emerging field of nanotube biotechnology. Nat. Rev. Drug Discov. 2, 29-37 (2003)

2. Bechet, D., Couleaud, P., Frochot, C., Viriot, M.L., Guillemin, F., Barberi-Heyob, M.: Nanoparticles as vehicles for delivery of photodynamic therapy agents. Trends Biotechnol. 26, 612-621 (2008)

3. Ulijn, R.V., Smith, A.M.: Designing peptide based nanomaterials. Chem. Soc. Rev. 37, 664-675 (2008)

4. Kotov, N.A., et al.: Nanomaterials for neural interfaces. Adv. Mater. 21, 3970-4004 (2009)

5. Yang, W.R., Ratinac, K.R., Ringer, S.P., Thordarson, P., Gooding, J.J., Braet, F.: Carbon nanomaterials in biosensors: should you use nanotubes or graphene? Angew. Chem. Int. Ed. 49, 2114-2138 (2010)

6. Wang, Y., Li, Z., Wang, J., Li, J., Lin, Y.: Graphene and graphene oxide: biofunctionalization and applications in biotechnology. Trends Biotechnol. 29, 5 (2011)

7. Bao, H., Pan, Y., Li, L.: Recent advances in graphene-based nanomaterials for biomedical applications. Nano Life (2012). doi:10.1142/S179398441100030X

8. Chung, C., Kim, Y.K., Shin, D., Ryoo, S.R., Hong, B.H., Min, D.H.: Biomedical applications of graphene and graphene oxide. Acc. Chem. Res. (2013). doi:10.1021/ar300159f

9. Liu, Z., Robinson, J.T., Sun, X.M., Dai, H.J.: PEGylated nanographene oxide for delivery of water insoluble cancer drugs. J. Am. Chem. Soc. 130, 10876-10877 (2008)

10. Lu, C.H., Yang, H.H., Zhu, C.L., Chen, X., Chen, G.N.: A graphene platform for sensing biomolecules. Angew. Chem. Int. Ed. Engl. 48, 4785-4787 (2009)
11. Kim, H., Abdala, A.A., Macosko, C.W.: Graphene/polymer nanocomposites. Macromolecules 43, 6515-6530 (2010)

12. Kuilla, T., Bhadra, S., Yao, D., Kim, N.H., Bose, S., Lee, J.H.: Recent advances in graphene based polymer composites. Prog. Polym. Sci 35, 1350-1375 (2010)

13. Sun, X., Liu, Z., Welsher, K., Robinson, J., Goodwin, A., Zaric, S., Dai, H.: Nano-graphene oxide for cellular imaging and drug delivery. Nano Res. 1, 203-212 (2008)

14. Zhang, J., Yang, H., Shen, G., Cheng, P., Zhang, J., Guo, S.: Reduction of graphene oxide via L-ascorbic acid. Chem. Commun. 46, 112-114 (2010)

15. Fernandez-Merino, M.J., Guardia, L., Paredes, J.I., Villar-Rodil, S., Solis-Fernandez, P., Martinez-Alonso, A., et al.: Vitamin C is an ideal substitute for hydrazine in the reduction of graphene oxide suspension. J. Phys. Chem. C 114(14), 6426-6432 (2010)

16. Ma, J., Wang, X., Liu, Y., Wu, T., Liu, Y., Guo, Y., Li, R., Sun, X., Wu, F., Lia, C., Gao, J.: Reduction of graphene oxide with Llysine to prepare reduced graphene oxide stabilized with polysaccharide polyelectrolyte. J. Mater. Chem. A 1, 2192-2201 (2013)

17. Jayanthi, P., Lalitha, P., Shubashini, K.S.: Phytochemical investigation of the extracts of Eichhornia crassipes and its solvent fractionates. J. Pharm. Res. 4(5), 1405-1406 (2011)

18. Firdhouse, M.J., Lalitha, P.: Phyto-mediated synthesis of gold nanoparticles using the aqueous extract of Eichhornia crassipes (Mart.) Solms. J. Pharm. Res 1(5), 558-562 (2013)

19. Meo, M.: Proceedings of 9th International Conference on Composite Science and Technology Composite Science and Technology: 2020-Scientific and Technical Challenges, p. 1053. DEStech Publication, Lancaster (2013)

20. Li, M., Liu, C., Cao, H., Zhang, Y.: Surface charge research of graphene oxide, chemically reduced graphene oxide and thermally exfoliated graphene oxide. Adv. Mater. Res. 716, 127-131 (2013) 